\author{
Military Technical College \\ Kobry El-Kobbah, \\ Cairo, Egypt.
}

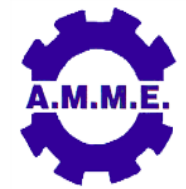
$17^{\text {th }}$ International Conference on Applied Mechanics and Mechanical Engineering.

\title{
THE IMPORTANCE OF ADDITIVE MANUFACTURING PROCESSES IN INDUSTRIAL APPLICATIONS
}

\author{
M. Al-Makky and D. Mahmoud ${ }^{* *}$
}

\begin{abstract}
Additive Manufacturing (AM) has evolved from just manufacturing prototypes into a process that manufacture high quality end parts in just a few years. The implementation of AM has significantly affected the medical, aerospace, automotive and tooling design applications. Due to several reasons, the production of medical implants using AM is highly emerging. Most importantly because the manufacturing of prosthesis and implants are case by case dependent. AM gives the benefit for those medical parts to be tailored according to patient's requirements. As for aerospace and automotive, the main target is to improve efficiency by decreasing fuel consumption. The role of AM in this industry is to reduce production lead time, reduce operational cost and build parts that have light weight. It is important to present a SWOT analysis, describing the strengths, weaknesses, opportunities and threats facing AM. There is a need to highlight the necessity for a standard practice to develop the AM process in the Egyptian market. Future trends to overcome current barriers are discussed in this work, point pinning on the development direction. Despite all the challenges that still faces AM, it is definitely emerging and expected to develop even more and take the lead in manufacturing techniques in the near future.
\end{abstract}

\section{KEY WORDS}

Additive Manufacturing, industrial applications, SWOT analysis.

Professor, Production Engineering Department, Faculty of Engineering, Alexandria University, Egypt, m.almakky@alexu.edu.eg.

** Graduate student, Production Engineering Department, Faculty of Engineering, Alexandria University Egypt, dalia.nabil@alexu.edu.eg. 


\section{INTRODUCTION}

After 30 years of developments, Additive Manufacturing (AM) has become a mainstream manufacturing method. The term itself can be defined as the building up of parts by adding materials one layer at a time based on a computerized 3D solid model [1]. The main key difference between this method and other manufacturing methods is that AM has an "additive" nature rather than a "subtractive" one. For example conventional turning, drilling, milling and grinding operations remove material from the original part to achieve the required shape [2]. AM on the other hand is considered "Additive" since it doesn't require any material removal or waste, thus considered an environmental friendly process [3].

At early stages of the AM it was usually referred to in different range of terminologies [4], one of the early synonyms was Layer Manufacturing (LM), due to the nature of building up the part. Sometimes also referred to as Solid Freeform Manufacturing, because of its ability to fabricate complex shapes. A more common name also is Rapid Prototyping (RP), when it was connected then in producing only prototypes not end user products. The terms Rapid Tooling (RT) started appearing referring to the printing of tools and dies, leading to improved mechanical properties, better dimensional and surface accuracy and enhancement in speeds. Finally, the process was evolved into Rapid Manufacturing or Additive Manufacturing. A good definition combined AM and RM to be "the use of a computer aided design (CAD)-based automated additive manufacturing process to construct parts that are used directly as finished products or components" [5].

Fig.1 describes the timeline development of AM specifically in aerospace industries. The development of RP process to AM in the past 40 years is illustrated [6], it is clear that the growth in production scale appeared when the patents for Fused Deposition Modeling (FDM) and Selective Laser Sintering (SLS) expired.

This study describes the development of industrial applications of AM focusing mainly on three of the most important at the present time, medical, automotive and aerospace applications. Then a SWOT analysis; which is a useful technique used to evaluate strength, weakness, opportunities and threats involved in the process is presented. Finally, future trends focusing on how to overcome present challenges to achieve excellence in AM is described.

\section{DEVELOPMENT OF AM IN INDUSTRIAL APPLICATIONS}

AM has flourished considerably in the past few years in specific industries. The industrial markets is making a huge development in using AM, for example medical implants, automotive, aerospace tooling and power generation. To gauge the current manufacturing maturity of $A M$ it is useful to measure its applications against the Manufacturing Readiness Level (MRL). MRL is a quantitative measure used to assess the maturity of a given industry or technology, it consists of 10 levels. Each level characterizes the progress in the development of a technology, from the idea (level 1) to the full deployment of the product in multiple marketplaces and multiple applications (level 10). Fig.2 presents the measure of various industrial applications 
with the aid of MRI [7]. It appears from the figure that medical applications have now reached full rate production, followed by tooling applications. Aerospace have proved capable in operational environment, while automotive applications are only capable in simulated environment.

\section{Tooling}

Tools, dies, and molds, usually referred to as "tooling," are required to produce nearly all plastic and metal products in industries such as automotive, medical, aerospace, and consumer electronics. Nevertheless, tooling production is a time consuming, technically difficult, and expensive production process that requires specialized materials, labor, and manufacturing techniques. Despite the expense and difficulty of designing and manufacturing tooling, dies and molds often have limited in-service lifetimes due to harsh use conditions including large loads under elevated temperatures, thermal cycling, repeated/ reversed loading conditions, contact with corrosive materials, and continuous production schedules[8]. Thus AM techniques have been utilized in this industry for decades, most common techniques used in manufacturing is Selective Laser Sintering (SLS), Direct Metal Deposition (DMD), and Fused Deposition Modeling (FDM) [9].

By the aid of AM techniques, it becomes possible to integrate optimized tempering channels in the tool directly and under near-contour conditions during production. This means that heat can be dissipated much after and more evenly, while hotspots can be cooled on a targeted basis [4]. This leads to longer tool life and shape preserving, moreover the plastic product quality and dimensional accuracy of the manufactured parts is enhanced. Fig. 3 contrasts the difference in design between conventional methods of cooling channel manufacturing and AM design. Furthermore, the repair of damaged tool components is an application area in which AM techniques can really demonstrate its strengths. Instead of having to dispose of a worn-out tool and replacing it with a new one, the damaged areas can be repaired quickly using laser sintering. This enables manufacturers to benefit from a longer operating life, also reducing operating costs.

\section{Medical}

The ability to convert medical imaging data into solid objects has made medical teams direct AM research in implants and prosthesis applications. Implants are used to reconstruct bone damage or defects cause by trauma or disease. One benefit of AM is the ability to manufacture complex internal mesh structures and porous structure; this allows bone to grow into the implants for better integration. Also it enables the variation of density to match the stiffness of the surrounding bones. Fig. 4 illustrates an additively manufactured prototype tibial and femoral stem knee implant and a cranial implant that was custom made for a specific patient, research claim that the use of AM can result in easier and shorter surgeries [10].

Various AM methods are used to fabricate the dental replacements, most common techniques are usually Selective Laser Melting (SLM), Direct Metal Laser Sintering (DMLS) or SLS[11]. Although the procedure is time demanding, the fact that no 
excess material is wastes is very profitable for applications with expensive material. $\mathrm{AM}$ has also influenced the production of hearing aids, devices that require a high degree of customization for individual fit. Greater ease of regulatory approval for devices worn on the body than for implants has assisted widespread adoption of AMproduced hearing aids, with the result that AM accounts for $99 \%$ of those that are placed in the ear [12].

Fig.5 Illustrates a dental bridge manufactured using chrome and cobalt by the aid on DMLS, and a customized hearing aid shells produced by Starkey technology.

\section{Automotive}

AM has been deployed in automotive industry for decades, however it is usually used for the production of prototypes, or complex custom parts for luxurious or antique cars [13]. For specialty cars or low-volume production, AM can be economical for some parts. Applications include custom parts on luxury cars or replacement parts on antique cars. For example of Bentley Motors used AM to fabricate some custom interior components, such as bezels, that were subsequently covered in leather and other materials. Typically, Bentley has production volumes of less than 10,000 cars for a given model, so this qualifies as low production volume.

Companies and research institutes also have successfully applied AM techniques to manufacture functional components for racing vehicles. Vehicles for motorsports usually use lightweight alloys (e.g., titanium) and have highly complex structures and low production volumes [14], thus they may use AM more than passenger car industries. Fig.6 shows some examples for commercially produced parts for automotive industries. All three components have been manufactured using SLM; the result of using additive manufacturing is lighter weight, thus faster speed.

\section{Aerospace}

Due to the fact that aerospace companies are project oriented manufacturers, a lot of different components are produced in low quantities [15], thus the use of AM becomes more attractive in these aerospace industry. Since AM isn't suitable for mass production but are more convenient in small scale production. Just as in automotive applications the most important factor in aerospace applications is to consider the decrease of the automobile weight, while maintain its safety [16].

Current applications of AM in aerospace industry includes printing low volume complex components and spare parts in metals and plastics [17].Using AM to manufacture the insides of some parts can reduce their weight by up to 70 percent, which can save an airline millions of gallons of fuel every year. For example, General Electric jet engines used to have 20 separate pieces of metal welded together, to achieve a shape that efficiently mixes fuel and air in a fuel injector. Aircraft engine component shown in Fig.7 are built using DMLS, in Fig.7 (a) laser melts metal in precise location beginning with a single layer shown on left size. The fuel nozzle shown in Fig.7(b) and (c) were designed for the highest level of efficiency while minimizing weight using DMLS. 
Another example is given in a rocket engine injector is one of the most critical and expensive engine components in a launch vehicle, and typically takes up to a year to conventionally manufacture. The NASA-PWR team was able to additively manufacture it in four months while reducing costs by 70 percent [18]. The component successfully passed testing; it was manufactured using selective laser melting. The additively manufactured injector was assembled from two parts, whereas a similar injector manufactured by the conventional means had 115 parts.

\section{SWOT ANALYSIS}

The SWOT analysis is used in this study to better illustrate and evaluate the position of AM compared to other manufacturing methods. It is a useful technique to address the strengths, weaknesses, opportunities and threats facing AM in industry at present time. A summary of the SWOT analysis is presented in Table 1.

\section{Strengths}

For the past 20 years AM has been well established process, gaining a lot of advantages in various fields. One advantage is the production of complex shapes and geometries, setting itself free from any material removal constraints. The fact that the procedure uses no tools, saves cost when compared to conventional manufacturing machines. Traditionally designers were oriented towards the concept of "Design for Manufacturing", this meant that the design was tailored to fit machining processes limitations. Nowadays this concept is changed into "Manufacture for Design" [19].

The ability to create what is now called cellular material [20] is major advantage, which is the application of material only where it is needed in specific applications. Its effect on biomedical applications is that it allows for regrowth of bones and tissue, thus enhancing its functions. As for aerospace/automotive applications, it offers high strength accompanied by relatively low mass, also providing good energy absorption characteristics and good thermal and acoustic insulation properties.

Moreover components that have complex shapes and need high strength to weight ratio uses materials such as titanium alloys or nickel super alloys [14]. These materials are costly, time consuming and hard to manufacture, resulting in a waste of material when tried to manufacture by conventional means. The use of AM saves the material loss and save time and effort of conventional machining [21]. Lighter aerospace components decrease the overall weight of the aircraft, thus reducing the fuel consumption and the carbon foot print through its life [11].

By limiting both the preprocessing and the post processing steps compared to conventional methods, AM reduces costs and lead time effectively. The effect of using AM on the supply chain of an aeronautics industry was studied. Not only has AM proved to be more cost efficient, but also a decline in transportation costs, inventory carrying cost, part obsolescence cost, thus improving the supply chain [22]. Moreover the elimination of assembling efforts enhances the accuracy of the product. 
Since AM can build complex products as one part, the need for welding and clamping is removed [23].

\section{Weakness}

The fact that AM products is built layer by layer makes it prone to staircase error [24]. This error results in a considerably rough surface finish, thus needing to post processing operations [25]. Up till now AM are more suitable for customized products, it isn't fit for mass production, and thus will have a limit in automotive industry [26]. Since the time needed to build up the part is relatively still long compared to conventional machining. The cost of materials and its availability are still considered a weak point in AM [27], also the absence of standards for those materials.

Users are still in need of education with regards to the process design, parameters and standardization. Although the process is mature enough to produce end parts, output from different machine could vary in specifications. The lack of in-process control, results in low reproducibility and process stability drops. This lack of standardization makes AM progress in aerospace applications and medical applications slow.

\section{Opportunities}

If future research should work on overcoming present limitations, AM will be able to cover more commercial applications. New research areas should be oriented to the developing of materials [28], these materials must account for variety, strength composition and finishing procedures in order to increase the applications of AM in various fields.

Areas of current research, which may lead to future products include the additive manufacture of embedded electronics, batteries and intelligent materials [19], the 3D printing of human tissue cells and organs [20] and the additive manufacture of intelligent materials at an atomic level [21]. The integration of Computer Numerically Controlled (CNC) machines with AM leading into what is known as Hybrid Manufacturing Systems is becoming popular[29]. Combining high productivity machining with material deposition is beginning to gain traction in the repair spare parts on site.

\section{Threats}

The lack of an in-process monitoring is considered a potential threat for AM. That is because although infrared cameras can be used for microstructure prediction, it can't be used for the repair of defects such as pinholes/porosity, micro cracks, and segregation [14]. The challenges involved in the sensing of AM processes include a lack of access to the build chamber and the need for intensive computing power. The sensing of AM processes may require fast on site measurements of the temperature, 
cooling rate, and residual stress; the calibration of fast optical sensors for high accuracy measurements; and in-process monitoring of geometric.

Due to the rapid development of a wide variety of technologies associated with AM, there is a lack of a comprehensive set of design principles, manufacturing guidelines, and standardization of best practices [26]. Another major concern is that soon CAD files will become prone to piracy and intellectual property concerns, since they are now available in large number online sites [30]. Also the ethical concerns of misuse of AM into manufacturing guns. Issues with materials, accuracy, surface finish, and certification standards have further limited its use [31].

\section{FUTURE TRENDS}

According to Strategic Research Agenda [32] consultation document a road map has been suggested to list all recommendations and future trend in AM. As illustrated in Fig.9 three main goals are set. First the in-process monitoring and control and predictability. Second the advanced materials, hybrid processes and embedding of components. Finally the focus on production speeds and market growth.

Future trends are focusing on shifting the production from low volume to mass production, also working on enhancing accuracy. The development of hybrid manufacturing process will help to transits the process into a new phase, since post processes operations will be eliminated. Also the development of techniques to produce AM parts in-situ. The development of new materials for AM, such as amorphous materials, cermets and graphene. The development of nano-particulates and nano-fiber materials as well is being under consideration. As for the process, stability reproducibility between different AM machines should be studied. Physics based models should be used to understand and predict defects and materials properties.

Most experts agree that the lack of additive manufacturing (AM) standards is a key point holding AM from further development. There are several standardization attempts that aims to enhance the reliability of AM techniques [33]. A road map for standardization has been suggested by the AM platform as shown in Fig.10. The main three goals focused on certification for quality of life enhancing applications, energy saving applications, and general mechanical applications.

\section{CONCLUSION}

The fast pace of additive manufacturing technologies is placing it ahead of conventional manufacturing techniques. The transition of RP techniques to AM, has taken place in less than 40 years. Through this period various techniques has been developed and the application of AM has evolved into manufacturing end part products. The various and versatile applications that $A M$ are involved in prove the fact that it is on the right track of development. From the MRL measure it appears that AM might not be fit for a mass production industry like automotive, but it has been used as a major contributor in motorcycles, custom made cars and race cars. The ability to construct various structures having high weight to strength ratio, made 
its activity in aerospace industry increase giving it a high MRL level. The ability to construct porous lattice thus allowing bone to regrow is well suited for applications like medical implants, reaching the highest level in MRL. A SWOT analysis is used to summarize the challenges and opportunities facing AM in industrial applications. From the future trends and recommendations, it was clear that the improvement of process monitoring and the development of new materials will lead to further development. Egyptian researchers should tackle this technology, focusing on all influencing parameters, such as build orientation techniques, layer thickness, and the effect of the process on the mechanical properties of the product and on the gained quality (dimensional, geometrical and surface finish) of the built products. Although AM has a lot of limitations and barriers holding it from achieving its potentials, there is no doubt that a bright future awaits it.

\section{REFERENCES}

[1] T. Wohlers and T. Gornet, "History of additive manufacturing Introduction of non-SL systems Introduction of low-cost 3D printers," Wohlers Rep. 2011, pp. 1-23, 2011.

[2] C. Beyer, "Strategic Implications of Current Trends in Additive Manufacturing," J. Manuf. Sci. Eng., vol. 136, no. 6, p. 064701, 2014.

[3] S. H. Huang, P. Liu, A. Mokasdar, and L. Hou, "Additive manufacturing and its societal impact: a literature review," Int. J. Adv. Manuf. Technol., vol. 67, no. 58, pp. 1191-1203, 2013.

[4] Y. Zhai, D. a. Lados, and J. L. LaGoy, "Additive Manufacturing: Making Imagination the Major Limitation," Jom, vol. 66, no. 5, pp. 1-9, 2014.

[5] E. N. Hopkinson and P. M. Dickens, Rapid Manufacturing. John Wiley \& Sons, 2006.

[6] M. J. Cotteleer, "3D opportunity: Additive manufacturing paths to performance, innovation, and growth," 2014.

[7] Dr. Berhard Langefeld, "Market Opportunities and Potentials of Additive Manufacturing," 2014.

[8] W. R. Morrow, H. Qi, I. Kim, J. Mazumder, and S. J. Skerlos, "Environmental aspects of laser-based and conventional tool and die manufacturing," J. Clean. Prod., vol. 15, no. 10, pp. 932-943, 2007.

[9] Michael Siemer, "'Adding' to Your Tooling Options," Modern Machine Shop, 2013.

[10] D. L. E. Murr, "3D Printing: Opportunities, Challenges, and Policy Implications of Additive Manufacturing," 2015.

[11] J. Parthasarathy, B. Starly, and S. Raman, "A design for the additive manufacture of functionally graded porous structures with tailored mechanical properties for biomedical applications," J. Manuf. Process., vol. 13, no. 2, pp. 160-170, 2011.

[12] T. S. Douglas, "Additive manufacturing: From implants to organs," South African Med. J., vol. 104, no. 6, p. 408, 2014.

[13] S. L. N. Ford, "Additive Manufacturing Technology : Potential Implications for U . S . Manufacturing," J. Int. Commer. Econ., no. September, pp. 1-35, 2014.

[14] Y. Huang, M. C. Leu, J. Mazumder, and A. Donmez, "Additive Manufacturing: Current State, Future Potential, Gaps and Needs, and Recommendations," J. Manuf. Sci. Eng., vol. 137, no. 1, p. 014001, 2015. 
[15] B. Lyons, "Additive Manufacturing in Aerospace Examples and Research Outlook," Front. Eng., vol. 42, no. 1, pp. 13-19, 2012.

[16] K. V. Wong and A. Hernandez, "A Review of Additive Manufacturing," ISRN Mech. Eng., vol. 2012, pp. 1-10, 2012.

[17] J. Coykendall, M. Cotteleer, L. Holdowsky, and M. Mahto, "3D opportunity in Aerospace and Defense," 2014.

[18] K. Maxey, "3D Printed Rocket Blasts Off," Engineering.com, 2013.

[19] C. B. Williams, F. Mistree, and D. W. Rosen, "A Functional Classification Framework for the Conceptual Design of Additive Manufacturing Technologies," J. Mech. Des., vol. 133, no. 12, pp. 1-11, 2011.

[20] C. Chu, G. Graf, and D. W. Rosen, "Design for Additive Manufacturing of Cellular Structures," Comput. Aided. Des. Appl., vol. 5, no. 5, pp. 686-696, 2008.

[21] M. Aliakbari, "Additive Manufacturing: state-of-the-art, capabilities, and sample applications with cost analysis," no. June, 2012.

[22] S. Das, J. J. Beama, M. Wohlert, and D. L. Bourell, "Direct laser freeform fabrication of high performance metal components," Rapid Prototyp. J., vol. 4, no. 3, pp. 112-117, 1998.

[23] O. Diegel, "Additive Manufacturing: The New Industrial Revolution," 2011.

[24] N. Siraskar, R. Paul, and S. Anand, "Adaptive Slicing in Additive Manufacturing Process Using a Modified Boundary Octree Data Structure," J. Manuf. Sci. Eng., vol. 137, no. 1, pp. 1-11, 2014.

[25] M. Agarwala, D. Bourell, J. Beaman, H. Marcus, and J. Barlow, "Postprocessing of selective laser sintered metal parts," Rapid Prototyp. J., vol. 1, no. 2, pp. 36-44, 1995.

[26] W. Gao, Y. Zhang, D. Ramanujan, K. Ramani, Y. Chen, C. B. Williams, C. C. L. Wang, Y. C. Shin, S. Zhang, and P. D. Zavattieri, "The status, challenges, and future of additive manufacturing in engineering," Comput. Des., 2015.

[27] M. Ruffo and R. Hague, "Cost estimation for rapid manufacturing simultaneous production of mixed components using laser sintering," Proc. Inst. Mech. Eng. Part B J. Eng. Manuf., vol. 221, no. 11, pp. 1585-1591, 2007.

[28] C. Chen, J. L. Erkal, B. C. Gross, S. Y. Lockwood, and D. M. Spence, "Evaluation of 3D Printing and Its Potential Impact on Biotechnology and the Chemical Sciences," Anal. Chem., vol. 86, no. 7, pp. 3240-3253, 2014.

[29] R. Bott, "A Review of Hybrid Manufacturing," Igarss 2014, no. 1, pp. 1-5, 2014.

[30] E. Matias and B. Rao, "3D Printing: On Its Historical Evolution and the Implications for Business," pp. 551-558, 2015.

[31] B. D. H. Freedman, "Layer by Layer," Technol. Rev. MIT, vol. 2002, no. February, pp. 1-3, 2012.

[32] E. MUÑIZ, "Additive Manufacturing in FP7 and Horizon 2020," 2014.

[33] M. D. Monzón, Z. Ortega, a. Martínez, and F. Ortega, "Standardization in additive manufacturing: activities carried out by international organizations and projects," Int. J. Adv. Manuf. Technol., vol. 76, no. 5-8, pp. 1111-1121, 2015.

[34] T. Halterman, "Aerojet Rocketdyne Successfully Completes Hot-Fire Tests of 3D Printed Rocket Engine Injectors," 3D Design, 3D Printers, 3D Printing, 3D Printing Materials, 2015. 


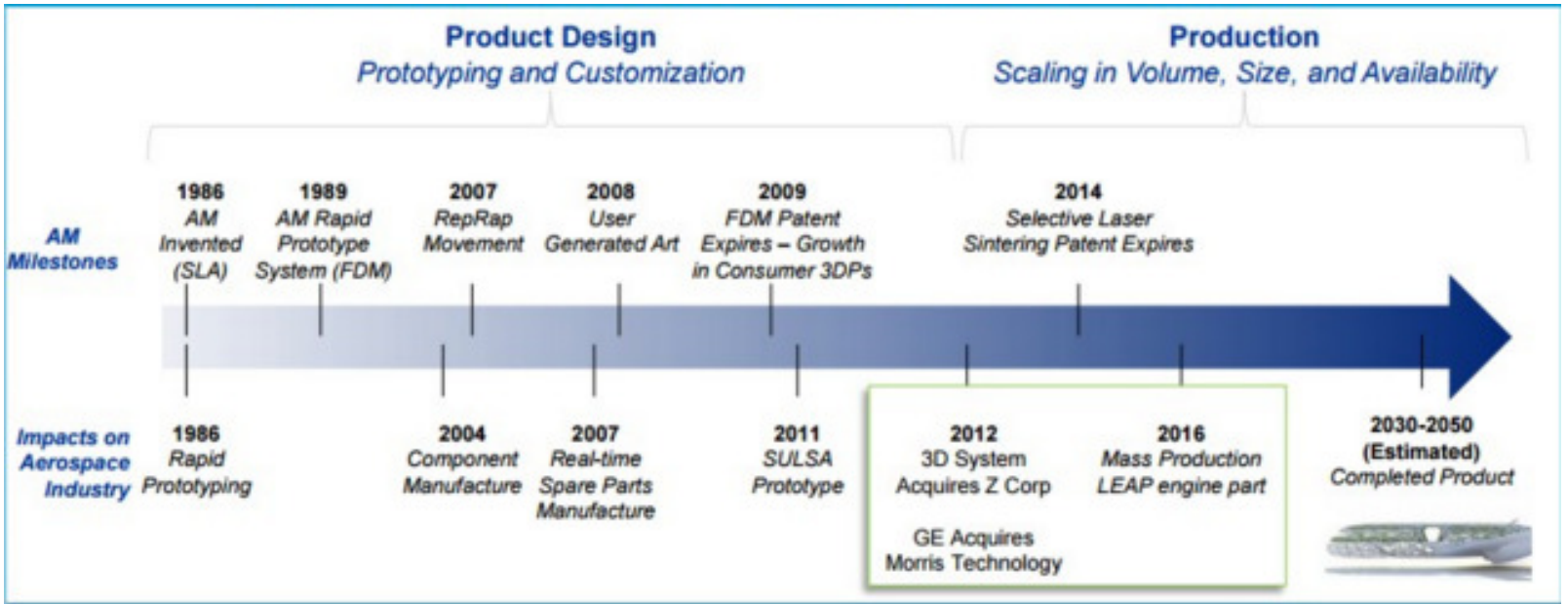

Fig.1. Historical Development of AM [6].

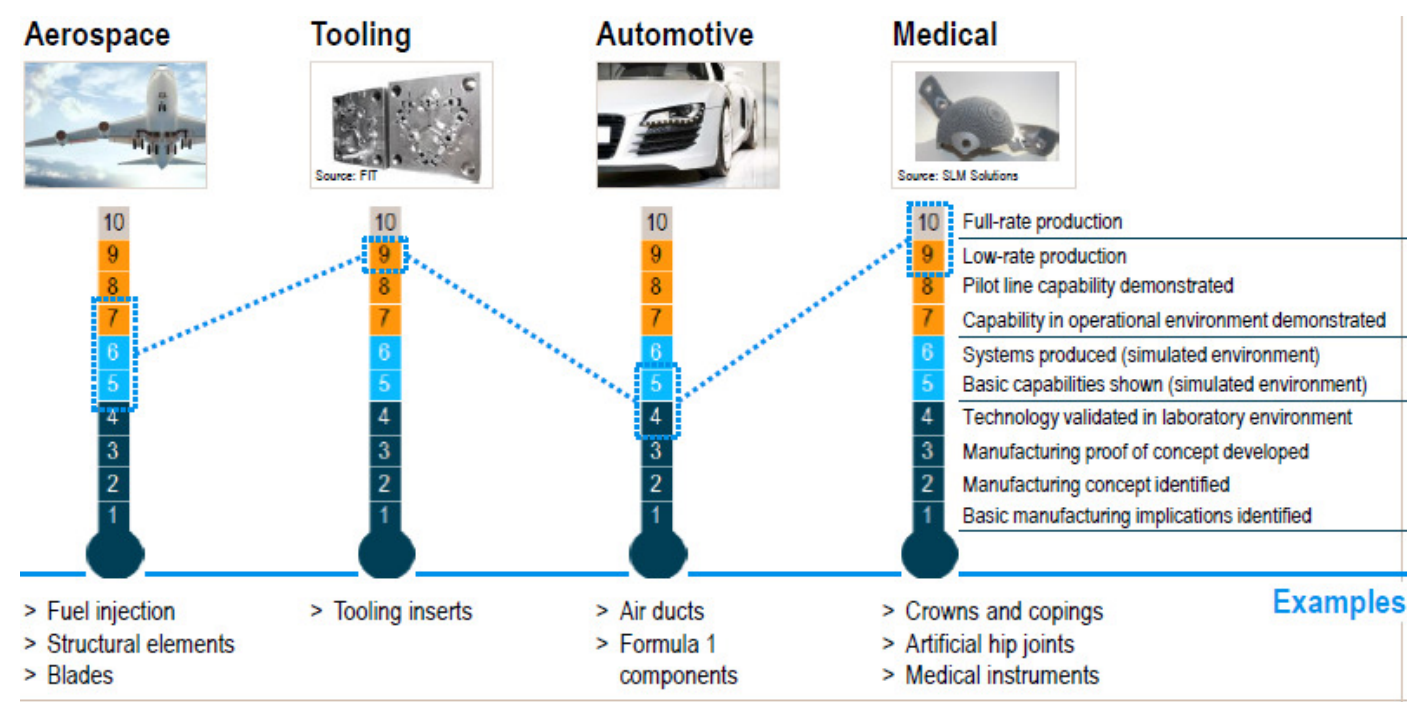

Fig.2. Industrial Applications Measures with the aid of MRL [7].

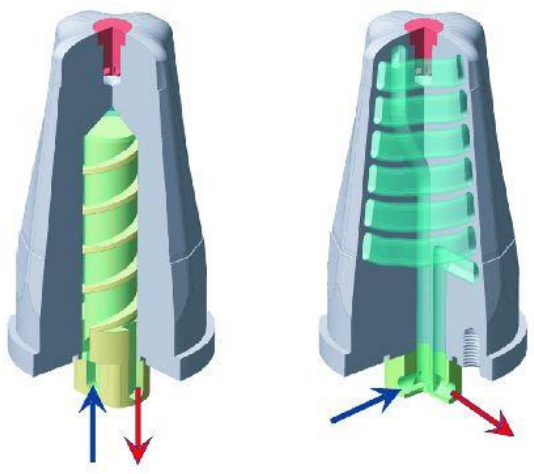

Fig.3. Conventional and AM cooling channels in a plastic cup die [9]. 

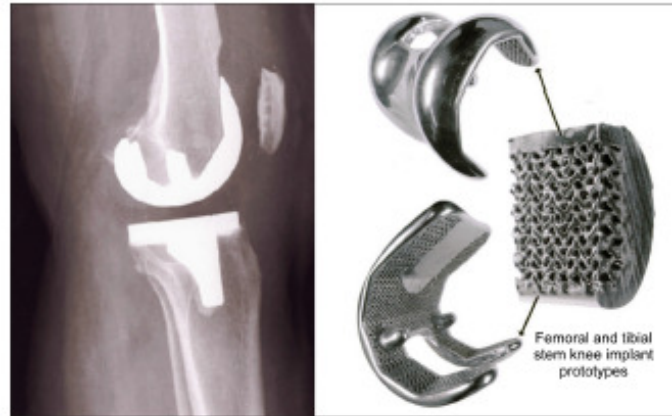

(a)
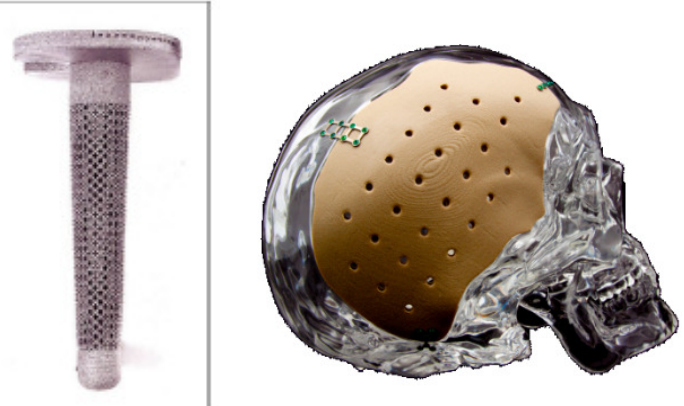

(b)

Fig.4. (a)Complex Internal Structure of a Knee Implant, (b) Custom Patient-specific Cranial Implant[10].

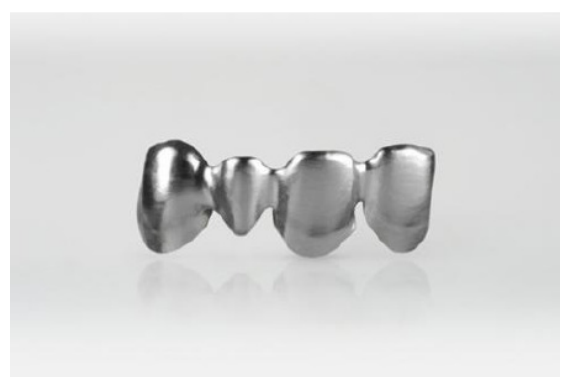

(a)

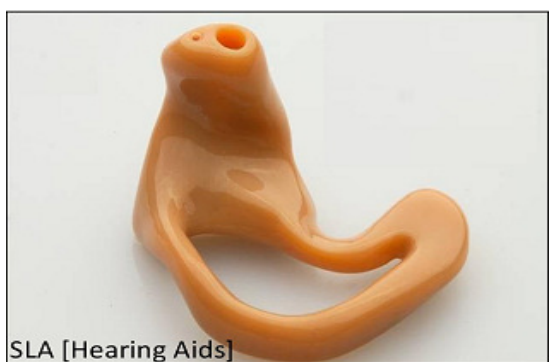

(b)

Fig.5. (a) Cobalt chrome polished dental bridge produced by DMLS [curtesy of EOS], (b) : Individually customized hearing aid shells [curtesy od StarKey® hearing technologies]

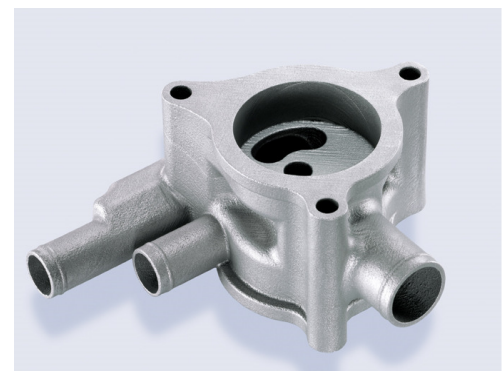

(a)

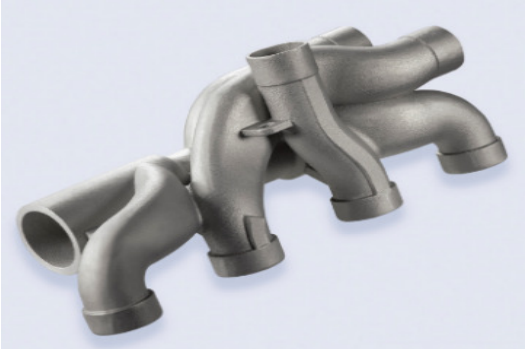

(b)

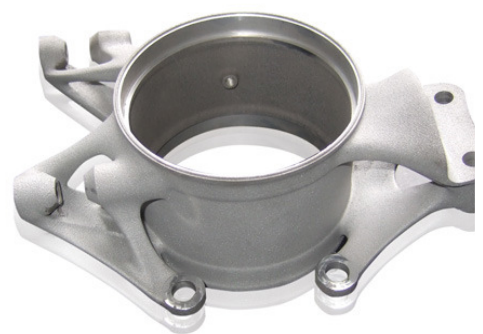

(c)

Fig.6. Examples of Automotive spare parts manufactured using DMLS (a) Oil pump housing [curtesy of Concept Laser](b) exhaust gas manifold [curtesy of Concept Laser](c) a Knuckles or an (axle pivot) [courtesy of EOS]. 


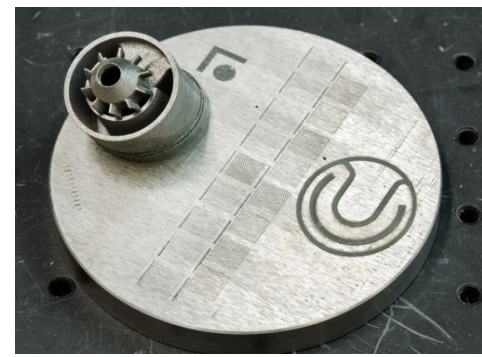

(a)

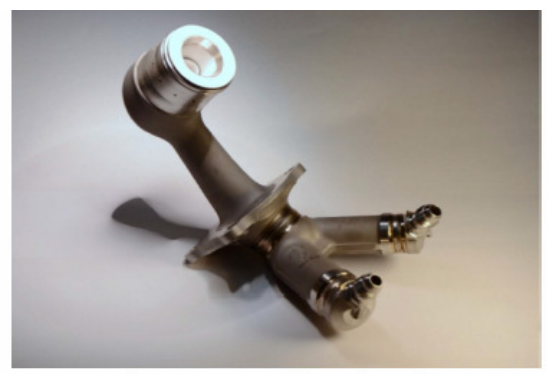

(b)

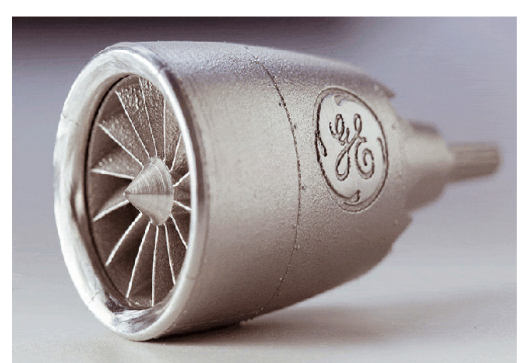

(c)

Fig.7. (a)GE made the aircraft engine component using DMLS, (b) GE jet engine Fuel Nozzle (c) GE's Jet Engine [31].

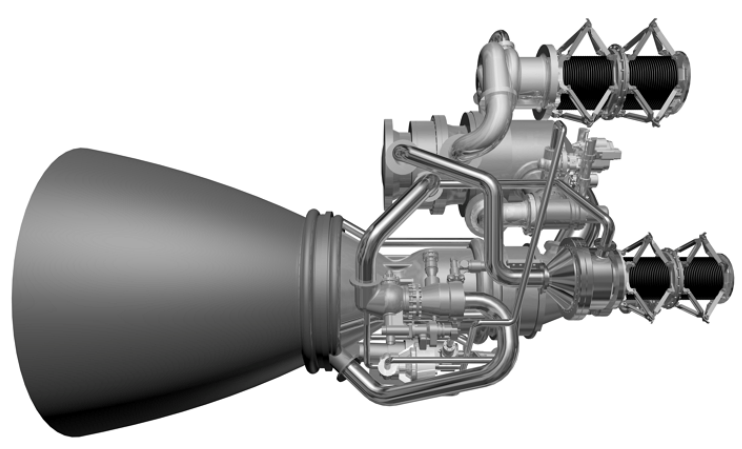

Fig.8. Rocket engine injector 3d model [34].

Table 1. SWOT analysis for AM technology.

\section{Strengths}

- Complex shapes and geometries

- No tooling needed

- Flexibility in changing design

- Customized products

- Reduce material waste compared to conventional methods

- Increased supply chain proficiency

\section{Weakness}

- Relatively poor surface finish

- post processing operations

- Requires knowledge of CAD software

- Not a wide range of materials available

- Relatively high costs of machinery and materials.

- Significantly slow fabrication speeds.

- Not fit for mass production

- Lack of repeatability

- The lack of multi-material deposition

\section{Threats}

- 3D printing of organs

- AM of intelligent material

- Nano-printing

- Developing new materials

- Extending applications to print spare parts directly in-situ.

- Improving Process sustainability
- The lack of in process monitoring.

- Reliability and standardization.

- Hype and unrealistic expectations.

- Ethical constraints ( gun printing)

- Cybersecurity risks ( CAD drawing piracy )

- Intellectual property/privacy issues 


\section{Roadmap for Technical AM Development}

Additive Manufacturing SRA 2013

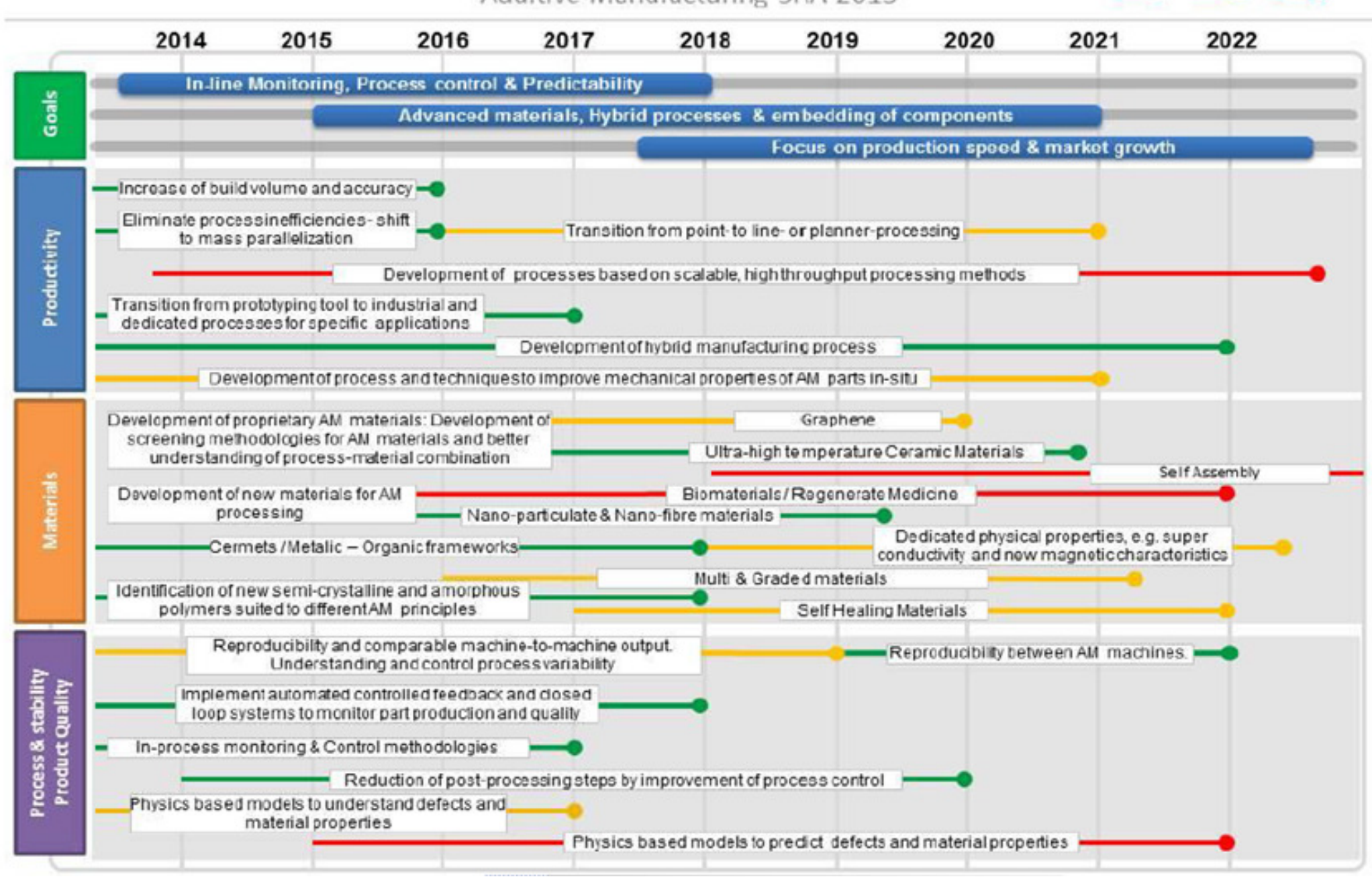

Fig.9. Road for Technical AM Development [32]. 


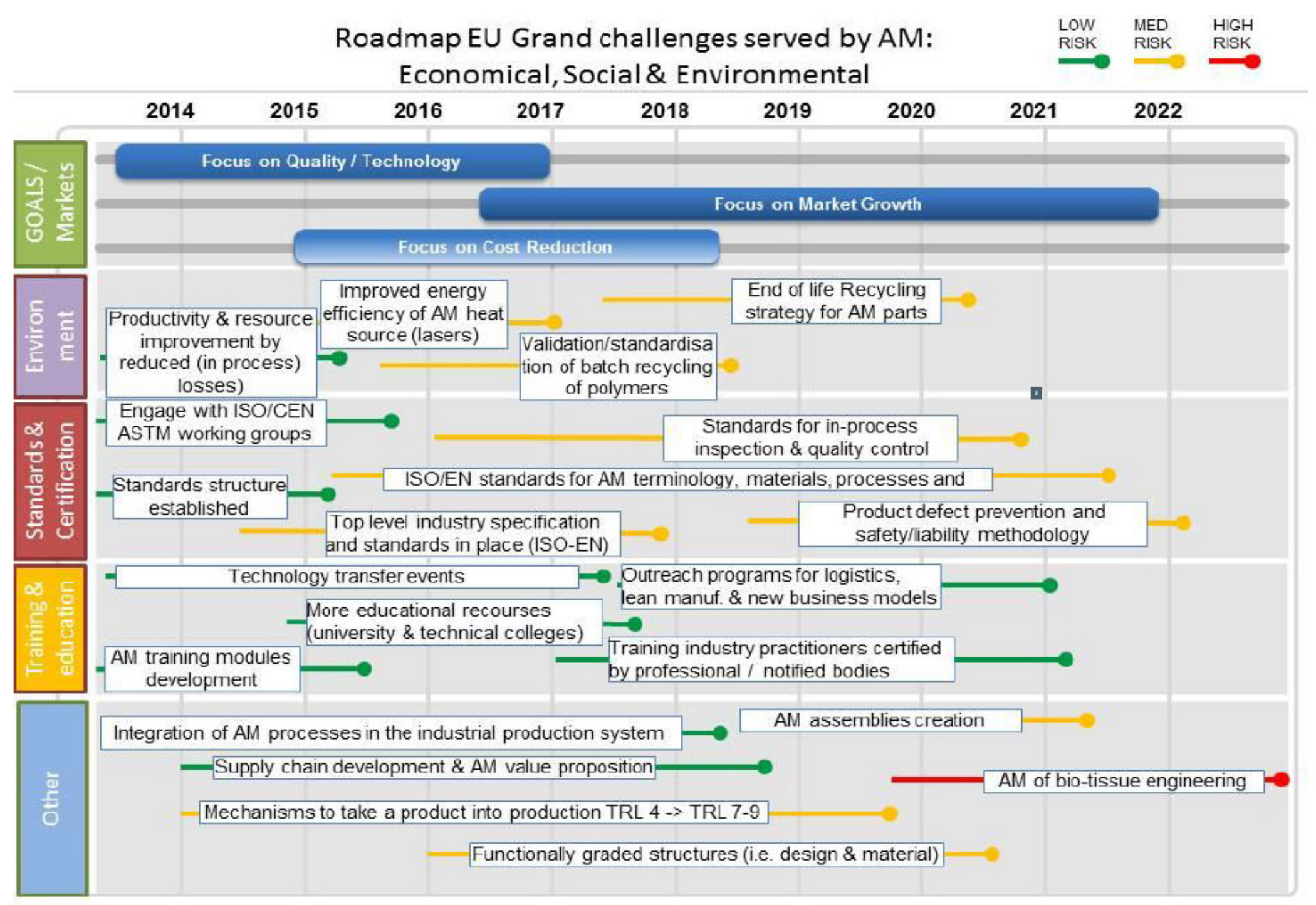

Fig.10. Timeline road map for standardization of AM [32] 